\title{
Non-standard and Numerov finite difference schemes for finite difference time domain method to solve one- dimensional Schrödinger equation
}

\author{
Lily Maysari Angraini and I Wayan Sudiarta* \\ Program Studi Fisika, Fakultas Matematika dan Ilmu Pengetahuan Alam, \\ Universitas Mataram, NTB, Indonesia \\ E-mail : * $\underline{\text { wayan.sudiarta@unram.ac.id }}$
}

Received 15 March 2018, Revised 29 March 2018, Published 31 March 2018

\begin{abstract}
The purpose of this paper is to show some improvements of the finite-difference time domain (FDTD) method using Numerov and nonstandard finite difference (NSFD) schemes for solving the one-dimensional Schrödinger equation. Starting with results of the unmodified FDTD method, Numerov-FD and NSFD are applied iteratively to produce more accurate results for eigen energies and wavefunctios. Three potential wells, infinite square well, harmonic oscillator and Poschl-Teller, are used to compare results of FDTD calculations. Significant improvements in the results for the infinite square potential and the harmonic oscillator potential are found using Numerov-NSFD scheme, and for Poschl-Teller potential are found using Numerov scheme.
\end{abstract}

Keywords: Finite difference time domain method, Time-dependent Schrodinger equations, Non-standard scheme, Numerov scheme

\section{Introduction}

The time-dependent Schrödinger equation is one of fundamental equations in quantum mechanics that is used for many problems in physics chemistry and the other natural sciences (Chen, Xu, \& Sun, 1993; Sullivan \& Citrin, 2001). Many methods have been developed to solve the time-dependent Schrodinger equation. Namely Monte Carlo (Winstead \& Ravaioli, 2003; Asparu-Guzik, William, \& Lester, 2003), DMRG (Chan \& Head-Gordon, 2003), Supersymmetri Quantum Mechanics (Koc \& Tutunculer, 2003), finite difference scheme (Simon \& Williams, 1999; Chen, Xu, \& Sun, 1993; Cooper, Valavanis, Ikonik, Harisson, \& Cunningham, 2010), non-standard finite difference scheme (Kalogiratou, Monovasilis, \& Simos, 2004), and finite difference time domain (Sudiarta \& Geldart, 2007; Sullivan \& Citrin, 2001). Each method focuses on speed and high numerical accuracy to determine the Schrodinger equation.

A well known class of method to solve the Schrodinger equation is finite difference scheme (FDS's). Chen et al (1993) was applied the finite difference schemes as a numerical solution of the Schrodinger equation. Simons and Wiliams (1999) used the finite difference scheme to solve the radial Schrodinger equation. Cooper et all (2010) 
studied the finite difference scheme to solve the Schrodinger equation with band non parabolicity in mid-infrared quantum cascade laser.

The another way to use the finite difference scheme for solving the Schrodinger equation is to modify the finite difference scheme using the nonstandard techniques (Mickens, 1999). The nonstandard finite difference scheme has a most important property is, in many cases, the method can eliminate the elementary numerical instability which plague the usual finite difference scheme (Mickens, 1999). Mickens and Ramadhani (1992) used the nonstandard techniques for constructing the finite difference model of differential equation. It was showed that the nonstandard finite differential scheme (NSFD) performs better than the standard method to solve the general case of finite potential range in radial Schrodinger equation (Kalogiratou, Monovasilis, \& Simos, 2004).

Recently, the finite difference time domain (FDTD) method has been applied for solving the Schrodinger equation (Sullivan \& Citrin, 2001). The FDTD method has been given for the accurate solution of the Schrodinger equation to determine the energies and the eigen function (Sudiarta, \& Geldart, 2007). Sudiarta and Geldart (2007) have used the FDTD method to compute the single density matrix particle. Sudiarta and Angraini (2016) have also applied the FDTD method with the symmetry quantum mechanics to obtain the ground and the excited state of particle in one dimensional (1D) potential. The FDTD method have been also used by Sudiarta and Angraini (2018) to determine energies and wave functions of two -electron quantum dots that was modeled by three dimensional (3D) oscillator harmonic potential. Subhan et all (2018) computed two particle thermal density matrices using finite difference time domain method.

Application of nonstandard finite difference time domain (NSFDTD) method is an alternative method to increase the speed and numerical accuracy of solving the Schrodinger equation. Sudiarta (2018) showed that the standard numerical scheme for a second derivative in spatial domain is replaced by a non-standard numerical scheme. It is shown the significant improvements using the NSFDTD method.

The other method which has the good performance to solve the Schrodinger equation with the high speed and accuracy is Numerov method. The Numerov method is an efficiency algorithm to determine the solution of second derivative (Gonzalez \& Thomposon, 1997). Kalogiratou et al (2004) is showed that the time-independent of the two dimensional Schrodinger depend on partial discretization. The partial discretization can be solved by differensial equation concept, and the Numerov method can be used to solve the problem. Pillai et al (2012) have used the matrix-Numerov to find the solution of Schrodinger equation, that it used to rekpresent the kinetic energy and discrete lattice. Chen et al (1993) have made the modification in the standard scheme with Numerov method to solve the Schrodinger equation solution, and the Numerov-finite difference scheme performs better than the standard method. Fack and Berghe (1987) is introduced an extended version of the well known Numerov method to obtain the numerical results for variety of potential with the high degree precision and accuracy. It is also found to be exact for the series expansion. 
Application of the FDTD method with Numerov method and Numerov - NSFDTD have not been done previously. In this paper, the Numerov method is used to modify the standard FDTD method and nonstandard FDTD method such that both of methods can be used for large spatial grid spacing.

This paper is organized as follows: the theory of the Numerov-FDTD method, Numerov-NSFDTD method, numerical results of eigen-energies for a particle in an infinite square well potential, oscillator harmonic, symmetric form of poschl teller potential and the conclusion.

\section{Theory}

The one dimensional time-independent Schrödinger equation (TISE) for a system with a particle in a potential well, $V(x)$, using atomic units $(\hbar=m=1)$ is given by

$$
\frac{\partial^{2} \psi(x)}{\partial x^{2}}=-2(E-V(x)) \psi(x)=-W(x) \psi(x)
$$

where $W(x)=2(E-V(x))$ and $E$ is the energy of the system. As shown by Sudiarta and Geldart (2007) that TISE can be solved by an iterative method which is derived from a finite difference scheme for a diffusion equation (or the time-dependent Schrödinger equation in imaginary time) given by

$$
\frac{\partial \psi(x, t)}{\partial t}=\frac{1}{2} \frac{\partial^{2} \psi(x, t)}{\partial x^{2}}-V(x) \psi(x, t)
$$

Starting with an arbitrary initial wave function $\psi(x, 0)$, Eq. (2) is used to evolve the wavefunction. After large interval of time, the wave function approaches the ground state wavefunction of the system. Excited states of the system can be determined by similar procedure provided that the wave function is orthogonal to lower energy wavefunctions.

After the wavefunctions are obtained, the energies are then computed by

$$
E=\frac{\int \psi^{*}(x) \widehat{H} \psi(x) d x}{\int|\psi(x)|^{2} d x}
$$

Because a finite computational domain is used, the outer computational boundary is terminated by a dirichlet boundary condition, $\psi(x=$ boundary, $t)=0$.

\section{Numerical Method}

A numerical procedure known as the FDTD method (Sudiarta \& Geldart, 2007) is applied to evolve the wavefunction using Eq. (2). In the FDTD method, the numerical discretization for the second derivative of Eq. (1) is the central finite difference (FD) scheme given by

$$
\left[\frac{\partial^{2} \psi(x)}{\partial x^{2}}\right]_{x=i \Delta x} \approx \frac{\psi(i+1)-2 \psi(i)+\psi(i-1)}{(\Delta x)^{2}}
$$

Where a notation $\psi(i) \equiv \psi(i \Delta x)$ is used and $\Delta x$ is the spatial grid spacing.

The accuracy of the FDTD method can be improved by modifying Eq. (4) using the non-standard FD scheme developed by Mickens (1992)[6] as follows 


$$
\left[\frac{\partial^{2} \psi(x)}{\partial x^{2}}\right]_{x=i \Delta x} \approx \frac{\psi(i+1)-2 \psi(i)+\psi(i-1)}{g(i)}
$$

For solving TISE, the appropriate function for $g(i) \equiv g(i \Delta x)=g(x)$ is given by (Mickens \& Ramadhani, 1992; Chen, Xu, \& Sun, 1993, Sudiarta, 2018)

$$
g(x)=\left\{\begin{array}{lll}
(\Delta x)^{2} & \text { for } & W(x)=0 \\
\frac{4}{W(x)} \sin ^{2}\left(\sqrt{W(x)} \frac{\Delta x}{2}\right) & \text { for } & W(x)>0 \\
\frac{4}{-W(x)} \sinh ^{2}\left(\sqrt{-W(x)} \frac{\Delta x}{2}\right) & \text { for } & W(x)<0
\end{array}\right.
$$

Besides the non-standard FD scheme, one can also use the Numerov finite difference scheme given by

$$
\left[\frac{\partial^{2} \psi(x)}{\partial x^{2}}\right]_{x=i \Delta x} \approx \frac{1}{(\Delta x)^{2}}\left[\begin{array}{c}
\psi(i+1)\left(1-\frac{h^{2}}{12} W(i+1)\right)-2 \psi(i)\left(1+\frac{h^{2}}{12} W(i)\right) \\
+\psi(i-1)\left(1-\frac{h^{2}}{12} W(i-1)\right)
\end{array}\right]
$$

As proposed by Chen et. al (1993), the non-standard FD and the Numerov FD schemes can be combined as shown in Eq. (8).

$$
\left[\frac{\partial^{2} \psi(x)}{\partial x^{2}}\right]_{x=i \Delta x} \approx \frac{\psi(i+1)\left(1-\frac{h^{2}}{12} W(i+1)\right)-2 \psi(i)\left(1+\frac{h^{2}}{12} W(i)\right)+\psi(i-1)\left(1-\frac{h^{2}}{12} W(i-1)\right)}{g(i)\left(1+\frac{h^{2}}{12} W(i)\right)}
$$

Following Sudiarta and Geldart (2007), an explicit iterative formula for the nonstandard and the Numverov FDTD is found to be

$$
\psi^{n+1}(i)=\alpha(i) \psi^{n}(i)+\beta(i)\left[\frac{\partial^{2} \psi(x)}{\partial x^{2}}\right]_{x=i \Delta x}
$$

with $\alpha(i)=[1-\Delta t V(i) / 2] /[1+\Delta t V(i) / 2]$ and $\beta(i)=\Delta t /[1+\Delta t V(i) / 2]$.

The energy of the system is then numerically determined by

$$
E=\frac{1}{\sum_{i}[\psi(i)]^{2}} \sum_{i}\left\{-\frac{1}{2} \psi(i)\left[\frac{\partial^{2} \psi(x)}{\partial x^{2}}\right]_{x=i \Delta x}+V(i) \psi(i)^{2}\right\}
$$

\section{Results and Discussion}

Three systems with three different potential wells are used to compare accuracies of the four FD schemes. The potentials are (1) infinite square well, (2) an harmonic oscillator potential and (3) a symmetric Poschl Teller potential.

\subsection{Infinite Square Well Potential}

Following Sudiarta (2018)[5], the simplest potential $V(x)=0$ for the interval of $0<$ $x<1$ and $V(x)=\infty$ for $x<0$ and $x>0$ is used. This is the one-dimensional box 
potential. The potential $V(x)=\infty$ can be achieved by setting the wavefunctions at the boundaries to zeros, $\psi(0)=0$ and $\psi(1)=0$.

Numerical results for eigen energies are shown in Table 1 for the parameters $\Delta x=$ 0.1 and $\Delta t=(\Delta x)^{2} / 10$. It is noted in Table 1 that NSFDTD and Numerov-NSFDTD produces the same as the exact results. The Numerov-FDTD gives better results than the FDTD but less accurate than the NSFDTD.

Table 1. Numerical eigen-energies for a particle in an infinite square potential computed by the FDTD method, the non-standard FDTD (NSFDTD) method, the Numerov-FDTD method and Numerov-NSFDTD (modified Numerov) are compared with exact results, $E_{n}=n^{2} \pi^{2} / 2$.

\begin{tabular}{lccccc}
\hline $\mathbf{n}$ & FDTD & NSFDTD & Numerov - FDTD & Numerov-NSFDTD & Exact \\
\hline 1 & 4.894348 & 4.934802 & 4.934601 & 4.934802 & 4.934802 \\
2 & 19.098301 & 19.739209 & 19.726195 & 19.739209 & 19.739209 \\
3 & 41.221475 & 44.413220 & 44.262411 & 44.413220 & 44.413220 \\
4 & 69.098301 & 78.956835 & 78.091632 & 78.956835 & 78.956835 \\
5 & 100.00000 & 123.37005 & 120.00000 & 123.37005 & 123.370055 \\
\hline
\end{tabular}

\subsection{Harmonic Oscillator Potential}

The second potential, also used by Sudiarta (2018), is an harmonic oscillator potential given $V(x)=\frac{1}{2} x^{2}$. The eigen energies for this case are $E_{n}=\left(n+\frac{1}{2}\right)$. Numerical eigenenergies for a grid spacing $\Delta x=0.8$ and a computational length of 16 are given in Table 2 .

Table 2. The eigen energies for an harmonic oscillator potential for four FD schemes are compared with the exact results.

\begin{tabular}{llrccc}
\hline $\mathbf{n}$ & FDTD & NSFDTD & Numerov - FDTD & Numerov-NSFDTD & Exact \\
\hline 0 & 0.479077 & 0.498019 & 0.498471 & 0.498430 & 0.500000 \\
1 & 1.391838 & 1.492586 & 1.489733 & 1.496356 & 1.500000 \\
2 & 2.188108 & 2.477781 & 2.459917 & 2.497042 & 2.500000 \\
3 & 2.954963 & 3.460975 & 3.406399 & 3.508486 & 3.500000 \\
4 & 3.236360 & 4.418670 & 4.182751 & 4.527097 & 4.500000 \\
\hline
\end{tabular}

\subsection{Symmetric Poschl Teller Potential.}

The third example of potentials is a symmetric Poschl Teller potential given by $V(x)=-\frac{\lambda(\lambda-1)}{2} \operatorname{sech}^{2}(x)$. For numerical calculations, $\lambda=5$ is used such that $V(x)=$ $-10 \operatorname{sech}^{2}(x)$. The energy states for this case are $E=-\frac{1}{2}(4-n)^{2}$ for $n \leq 4$. Comparisons for the numerical results of eigen energies with the exact energies are shown in Table 3 for grid parameters $\Delta x=0.5$ dan $N=80$. Similarly as in previous results that the NSFDTD, the Numerov-FDTD and the Numerov-NSFDTD methods are 
generally give more accurate results than the stan;dard FDTD. The accuracy of Numerov method is comparable with other non-standar schemes for this case except for third excited state where the Numerov method performs better. This indicates that the performance of FD schemes depend on the potential used.

Table 3. The eigenstate energies is for a symmetric Poschl Teller potential with computational parameters, grid spacing $\Delta x=0.5$ and number of grids $N=0.8$.

\begin{tabular}{rlrrrr}
\hline n & FDTD & NSFDTD & Numerov-FDTD & Numerov-NSFDTD & Exact \\
\hline 0 & -8.10688 & -8.02505 & -8.02395 & -8.02121 & -80 \\
1 & -5.01409 & -4.50844 & -4.51665 & -4.48821 & -4.5 \\
2 & -3.26980 & -2.13147 & -2.17337 & -2.04094 & -2.0 \\
3 & -1.34050 & -0.47285 & -0.48409 & -0.42118 & -0.5 \\
\hline
\end{tabular}

\section{Conclussion}

Three finite difference (FD) schemes are used to modify the standard finite difference time domain (FDTD) method. It has been shown that the non standard FDTD and Numerov-NSFDTD give generally more accurate results compared to the standard FDTD method. The Numerov-NSFDTD method is generally shown to perform better than the standard FDTD method for all cases.

\section{References}

Alan Asparu-Guzik, William A, and Lester Jr. (2003). Quantum Monte Carlo methods for the solution of the Schrodinger equation for moleculer systems. Handbook of Numerical Analysis, Elevesier. Doi: 10.1016/51570-8659(03)10007-5.

Chen, R., Xu, Z., \& Sun, L. (1993). Finite-difference scheme to solve Schrödinger equations. Physical Review E, 47(5), 3799.

Cooper, J. D., Valavanis, A., Ikonić, Z., Harrison, P., \& Cunningham, J. E. (2010). Finite difference method for solving the Schrödinger equation with band nonparabolicity in mid-infrared quantum cascade lasers. Journal of Applied Physics, 108(11), 113109. Doi : 10.1063/1.3512981.

Garnet Kin-Lic Chan, and Martin Head-Gordon. (2003). Exact solution (within a triplezeta, double polarization basis set) of the electronic Schrodinger equation for water. J.Chem.Phys. Doi : 10.1063/1.1574318.

Kalogiratou, Z. Monovasilis, Th. Simos, T.E. (2004). Numerical solution of the twodimensional time independent Schrodinger equation with Numerov-type methods. Journal of Mathematical Chemistry Vol. 37, No 3. Doi: 10.1007/s10910-004-1469-1.

Mohandas Pillai, Joshua Goglio, And Thad G. Walker. (2012). Matrix Numerov method for solving Schrodinger's equation. Am. J. Phys. 80(11) : 1017. https://dx.doi.org/10.1119/1.4748813.

Quiroz González, J. L. M., \& Thompson, D. (1997). Getting started with Numerov's method. Computers in Physics, 11(5), 514-515.

Ramazan Koc and Hayriye Tutunculer. (2003). Exact Solution of position dependent 
mass Schrodinger equation by Supersymmteric quantum mechanics. Ann.Phys. (Leipzig). Doi: 10.1002/andp200310031.

Ronald E Mickens, Issa Ramadhani. (1992). Finite-difference scheme for the numerical solution of the Schrodinger equation. Physical Review A, 45(3) : 2074. https://doi.org/10.1088/0031-8949/81/04/045001

Ronald E. Mickens. (1999). Application of Nonstandard Finite Difference Schemes. World Scientific Publishing, Singapore. ISBN : 981-02-4133-X.

Simon, T.E. Williams, P.S. (1999). On finite difference methods for the solution of the Schrodinger equation. Computer \& Chemistry, 23 (513-554). Doi: S0097$\underline{8485(99) 00023-6 .}$

Subhan, I Wayan Sudiarta, and Lily Maysari Angraini. (2018). Two-Particle Thermal Density Matrices in One Dimension Using Finite Difference Time Domain (FDTD) Method. Indonesian Physical Review. Doi :10.29303/ipr.vlil.13.

Sudiarta, I. W., \& Geldart, D. W. (2007). Solving the Schrödinger equation using the finite difference time domain method. Journal of Physics A: Mathematical and Theoretical, 40(8), 1885. https://doi.org/10.1088/1751-8113/40/8/013

Sudiarta, I. W., \& Angraini, L. M. (2016). Determination of excited states of quantum systems by finite difference time domain method (FDTD) with supersymmetric quantum mechanics (SUSY-QM). In AIP Conference Proceedings (Vol. 1729, No. 1, p. 020030). AIP Publishing. doi: 10.1063/1.4946933.

Sudiarta, I. W., \& Angraini, L. M. (2018, October). The finite difference time domain (FDTD) method to determine energies and wave functions of two-electron quantum dot. In AIP Conference Proceedings (Vol. 2023, No. 1, p. 020199). AIP Publishing. Doi : $10.1063 / 1.5064196$

Sudiarta, I. W. (2018). Non-standard finite-difference time-domain method for solving the Schrödinger equation. Pramana, 91(4), 52. https://doi.org/10.1007/s12043018-1632-Z

Sullivan, D., \& Citrin, D. S. (2001). Time-domain simulation of two electrons in a quantum dot. Journal of Applied Physics, 89(7), 3841-3846.

Veerle Fack and G Vanden Berghe. (1987). (Extended) Numerov Method for Computing Eigenvalues of Specific Schrodinger Equation. Journal of Physics A: Mathematical and General. Doi : 10.1088/0305-4470/20/13/022.

Winstead, B., \& Ravaioli, U. (2003). A quantum correction based on Schrodinger equation applied to Monte Carlo device simulation. IEEE Transactions on Electron Devices, 50(2), 440-446. Doi : 10.1109/TED.2003.809431. 